\title{
Toward the existence of the odderon as a three-gluon bound state
}

\author{
Hua-Xing Chen $\oplus^{1,{ }^{1 *}}$ Wei Chen $\oplus^{2, \dagger}$ and Shi-Lin Zhu ${ }^{3,4,5, \$}$ \\ ${ }^{1}$ School of Physics, Southeast University, Nanjing 210094, China \\ ${ }^{2}$ School of Physics, Sun Yat-Sen University, Guangzhou 510275, China \\ ${ }^{3}$ School of Physics and State Key Laboratory of Nuclear Physics and Technology, Peking University, \\ Beijing 100871, China \\ ${ }^{4}$ Collaborative Innovation Center of Quantum Matter, Beijing 100871, China \\ ${ }^{5}$ Center of High Energy Physics, Peking University, Beijing 100871, China
}

(Received 5 April 2021; accepted 22 April 2021; published 20 May 2021)

\begin{abstract}
Inspired by the evidence of the odderon exchange recently observed by the D0 and TOTEM Collaborations, a quantum chromodynamics (QCD) sum rule investigation is performed to study the odderon as a three-gluon bound state. There may exist six lowest-lying three-gluon odderons with the quantum numbers $J^{P C}=1 / 2 / 3^{ \pm-}$. We systematically construct their interpolating currents, and calculate their mass spectra. To verify their existence, we propose to search for the spin-3 odderons in their $V V V$ and $V V P$ decay channels directly at LHC, with $V$ and $P$ light vector and pseudoscalar mesons respectively.
\end{abstract}

DOI: 10.1103/PhysRevD.103.L091503

\section{INTRODUCTION}

Very recently, the D0 and TOTEM Collaborations compared their $p p$ and $p \bar{p}$ [1] cross sections, and found they differ with a significance of $3.4 \sigma$ [2]. This leads to the evidence of a $t$-channel exchanged odderon $[3,4]$, i.e., a colorless $C$-odd gluonic compound. They further combined their previous result of Ref. [5], and increased the significance of this evidence to 5.2-5.7 $\sigma$. Accordingly, the D0 and TOTEM Collaborations claimed that they have accomplished the first experimental observation of the odderon.

Actually, in the previous TOTEM experiment [5] they had attempted to probe the existence of the odderon, and there had been some discussions about it [6-9]. However, these discussions are mainly on the contribution of the odderon exchange in certain collisions, so it is crucial and important to directly study the odderon itself. Recall that the odderon was first introduced in 1973 [10], and later reintroduced in quantum chromodynamics (QCD) in the 1980s [11-13]. There have been several attempts to prove its existence, and we refer to the review in [14] for detailed discussions.

The odderon is predominantly a $C$-odd three-gluon bound state that is one category of glueballs. Glueballs

\footnotetext{
*hxchen@seu.edu.cn

†chenwei29@mail.sysu.edu.cn

zhusl@pku.edu.cn
}

Published by the American Physical Society under the terms of the Creative Commons Attribution 4.0 International license. Further distribution of this work must maintain attribution to the author(s) and the published article's title, journal citation, and DOI. Funded by SCOAP. are important for the understanding of nonperturbative QCDs, but there is currently no definite experimental evidence for their existence. There have been many discussions about them using various theoretical methods and models, such as the MIT bag model $[15,16]$, the flux tube model [17], the Coulomb gauge model [18,19], lattice QCD [20-24], QCD sum rules [25-28], etc. Mass spectra of the spin-0 odderons were systematically studied in Refs. [29-33] using QCD sum rules. However, in Ref. [34] the authors studied glueball trajectories, and their results suggest that the odderon is essentially an object in the Regge pole model, and any derivation of its finite mass from massless gluons meets conceptual difficulties in QCD, preventing reliable calculations of its spectrum.

The lowest-lying two, three, and four glueballs were systematically investigated in Ref. [35], where the authors classified altogether six lowest-lying odderons with the quantum numbers $J^{P C}=1 / 2 / 3^{ \pm-}$, and constructed their corresponding nonrelativistic low-dimension operators. Some of these operators have been successfully used in lattice QCD calculations. This inspired us to construct their corresponding relativistic odderon currents, which can be further applied to perform QCD sum rule analyses and calculate masses of the $J^{P C}=1 / 2 / 3^{ \pm-}$odderons. This is the aim of this article. Comparison of our QCD sum rule results with the lattice QCD results [21-24] can be found in Table II.

\section{RELATIVISTC ODDERON CURRENTS}

As the first step, we use the gluon field strength tensor $G_{\mu \nu}^{a}$ to construct relativistic odderon currents. Here $a$ is a color index; $\mu$ and $\nu$ are Lorentz indices. Additionally, we 
use $d^{a b c}$ to denote the totally symmetric $S U_{c}(3)$ structure constant, and $\tilde{G}_{\mu \nu}^{a}$ to denote the dual gluon field strength tensor, defined as $\tilde{G}_{\mu \nu}^{a}=G^{a, \rho \sigma} \times \epsilon_{\mu \nu \rho \sigma} / 2$.

In Ref. [35] the chromoelectric and chromomagnetic fields $(i, j=1,2,3)$,

$$
E_{i}=G_{i 0} \quad \text { and } \quad B_{i}=-\frac{1}{2} \epsilon_{i j k} G^{j k}
$$

were used to write down the nonrelativistic low-dimension odderon operators:

$$
\begin{aligned}
& 1^{+-} d^{a b c}\left(\vec{E}_{a} \cdot \vec{E}_{b}\right) \vec{B}_{c}, \\
& 1^{--} d^{a b c}\left(\vec{E}_{a} \cdot \vec{E}_{b}\right) \vec{E}_{c}, \\
& 2^{+-} d^{a b c} \mathcal{S}\left[E_{a}^{i}\left(\vec{E}_{b} \times \vec{B}_{c}\right)^{j}\right], \\
& 2^{--} d^{a b c} \mathcal{S}\left[B_{a}^{i}\left(\vec{E}_{b} \times \vec{B}_{c}\right)^{j}\right], \\
& 3^{+-} d^{a b c} \mathcal{S}\left[B_{a}^{i} B_{b}^{j} B_{c}^{k}\right], \\
& 3^{--} d^{a b c} \mathcal{S}\left[E_{a}^{i} E_{b}^{j} E_{c}^{k}\right],
\end{aligned}
$$

where $\mathcal{S}$ denotes symmetrization and subtracting trace terms in the set $\{i j\}$ or $\{i j k\}$.

To perform QCD sum rule analyses, we further construct their corresponding relativistic currents:

$$
\begin{gathered}
J_{1}^{\alpha \beta}=d^{a b c} G_{a}^{\mu \nu} G_{b, \mu \nu} G_{c}^{\alpha \beta}, \\
J_{2}^{\alpha_{1} \alpha_{2}, \beta_{1} \beta_{2}}=d^{a b c} \mathcal{S}^{\prime}\left[G_{a}^{\alpha_{1} \beta_{1}} \tilde{G}_{b}^{\alpha_{2} \mu} G_{c, \mu}^{\beta_{2}}-\left\{\alpha_{2} \leftrightarrow \beta_{2}\right\}\right], \\
J_{3}^{\alpha_{1} \alpha_{2} \alpha_{3}, \beta_{1} \beta_{2} \beta_{3}}=d^{a b c} \mathcal{S}^{\prime}\left[G_{a}^{\alpha_{1} \beta_{1}} G_{b}^{\alpha_{2} \beta_{2}} G_{c}^{\alpha_{3} \beta_{3}}\right],
\end{gathered}
$$

where $\mathcal{S}^{\prime}$ denotes symmetrization and subtracting trace terms in the two sets $\left\{\alpha_{1} \cdots \alpha_{J}\right\}$ and $\left\{\beta_{1} \cdots \beta_{J}\right\}$ simultaneously.

The first current $J_{1}^{\alpha \beta}$ contains both $J^{P C}=1^{+-}$and $1^{--}$ components, and it couples to the $1^{+-}$and $1^{--}$odderons simultaneously:

$$
\begin{gathered}
\left\langle 0\left|J_{1}^{\alpha \beta}\right| X_{1^{+-}}\right\rangle=i f_{1^{+-}} \epsilon^{\alpha \beta \mu \nu} \epsilon_{\mu} p_{\nu}, \\
\left\langle 0\left|J_{1}^{\alpha \beta}\right| X_{1^{--}}\right\rangle=i f_{1^{--}}\left(p^{\alpha} \epsilon^{\beta}-p^{\beta} \epsilon^{\alpha}\right),
\end{gathered}
$$

where $f_{1^{+-}}$and $f_{1^{--}}$are decay constants, and $\epsilon_{\mu}$ is the polarization vector. This current has a partner

$$
\tilde{J}_{1}^{\alpha \beta}=d^{a b c} \tilde{G}_{a}^{\mu \nu} \tilde{G}_{b, \mu \nu} \tilde{G}_{c}^{\alpha \beta},
$$

which just has the opposite couplings:

$$
\begin{gathered}
\left\langle 0\left|\tilde{J}_{1}^{\alpha \beta}\right| X_{1^{+-}}\right\rangle=i f_{1^{+-}}\left(p^{\alpha} \epsilon^{\beta}-p^{\beta} \epsilon^{\alpha}\right), \\
\left\langle 0\left|\tilde{J}_{1}^{\alpha \beta}\right| X_{1^{--}}\right\rangle=i f_{1^{--}} \epsilon^{\alpha \beta \mu \nu} \epsilon_{\mu} p_{\nu^{\prime}} .
\end{gathered}
$$

Therefore, we can use either $J_{1}^{\alpha \beta}$ or $\tilde{J}_{1}^{\alpha \beta}$ to investigate the $1^{+-}$and $1^{--}$odderons at the same time. In the present study we use $J_{1}^{\alpha \beta}$ to investigate the $1^{+-}$odderon and $\tilde{J}_{1}^{\alpha \beta}$ to investigate the $1^{--}$one, because the couplings given in Eqs. (6) and (10) can be more easily calculated.

The currents $J_{2}^{\alpha_{1} \alpha_{2}, \beta_{1} \beta_{2}}$ and its partner

$$
\tilde{J}_{2}^{\alpha_{1} \alpha_{2}, \beta_{1} \beta_{2}}=d^{a b c} \mathcal{S}^{\prime}\left[\tilde{G}_{a}^{\alpha_{1} \beta_{1}} G_{b}^{\alpha_{2} \mu} \tilde{G}_{c, \mu}^{\beta_{2}}-\left\{\alpha_{2} \leftrightarrow \beta_{2}\right\}\right],
$$

couple to the $2^{+-}$and $2^{--}$odderons through:

$$
\begin{aligned}
\left\langle 0\left|J_{2}^{\cdots}\right| X_{2^{+-}}\right\rangle & =i f_{2^{+-}} \mathcal{S}^{\prime}\left[\epsilon^{\alpha_{i} \beta_{i} \mu_{i} \nu_{i}} \epsilon_{\mu_{i}} p_{\nu_{i}}\right]^{2}, \\
\left\langle 0\left|\tilde{J}_{2}^{\cdots}\right| X_{2^{--}}\right\rangle & =i f_{2^{--}} \mathcal{S}^{\prime}\left[\epsilon^{\alpha_{i} \beta_{i} \mu_{i} \nu_{i}} \epsilon_{\mu_{i}} p_{\nu_{i}}\right]^{2},
\end{aligned}
$$

where

$$
[\cdots]^{J}=\epsilon^{\alpha_{1} \beta_{1} \mu_{1} \nu_{1}} \epsilon_{\mu_{1}} p_{\nu_{1}} \cdots \epsilon^{\alpha_{J} \beta_{J} \mu_{J} \nu_{J}} \epsilon_{\mu_{J}} p_{\nu_{J}} .
$$

The current $J_{3}^{\alpha_{1} \alpha_{2} \alpha_{3}, \beta_{1} \beta_{2} \beta_{3}}$ and its partner

$$
\tilde{J}_{3}^{\alpha_{1} \alpha_{2} \alpha_{3}, \beta_{1} \beta_{2} \beta_{3}}=d^{a b c} \mathcal{S}^{\prime}\left[\tilde{G}_{a}^{\alpha_{1} \beta_{1}} \tilde{G}_{b}^{\alpha_{2} \beta_{2}} \tilde{G}_{c}^{\alpha_{3} \beta_{3}}\right]
$$

couple to the $3^{+-}$and $3^{--}$odderons through:

$$
\begin{aligned}
\left\langle 0\left|J_{3}^{\cdots}\right| X_{3^{+-}}\right\rangle & =i f_{3^{+-}} \mathcal{S}^{\prime}\left[\epsilon^{\alpha_{i} \beta_{i} \mu_{i} \nu_{i}} \epsilon_{\mu_{i}} p_{\nu_{i}}\right]^{3}, \\
\left\langle 0\left|\tilde{J}_{3}^{\cdots}\right| X_{3^{--}}\right\rangle & =i f_{3^{--}} \mathcal{S}^{\prime}\left[\epsilon^{\alpha_{i} \beta_{i} \mu_{i} \nu_{i}} \epsilon_{\mu_{i}} p_{\nu_{i}}\right]^{3} .
\end{aligned}
$$

It is interesting to notice that all the above currents have $N=2 J$ Lorentz indices with certain symmetries, so that they can couple to the positive- and negative-parity odderons simultaneously. For example, the spin-2 current $J_{2}^{\alpha_{1} \alpha_{2}, \beta_{1} \beta_{2}}$ has four Lorentz indices, satisfying

$$
J_{2}^{\alpha_{1} \alpha_{2}, \beta_{1} \beta_{2}}=-J_{2}^{\beta_{1} \alpha_{2}, \alpha_{1} \beta_{2}}=-J_{2}^{\alpha_{1} \beta_{2}, \beta_{1} \alpha_{2}}=J_{2}^{\alpha_{2} \alpha_{1}, \beta_{2} \beta_{1}} .
$$

\section{QCD SUM RULE ANALYSES}

In this section we use the current $J_{1}^{\alpha \beta}$ as an example to perform QCD sum rule analyses, a method that has been widely applied in the study of hadron phenomenology $[36,37]$. We study the two-point correlation function

$$
\begin{aligned}
\Pi^{\alpha \beta, \alpha^{\prime} \beta^{\prime}}\left(q^{2}\right) & \equiv i \int d^{4} x e^{i q x}\left\langle 0\left|\mathbf{T}\left[J_{1}^{\alpha \beta}(x) J_{1}^{\alpha^{\prime} \beta^{\prime} \dagger}(0)\right]\right| 0\right\rangle \\
& =\left(g^{\alpha \alpha^{\prime}} g^{\beta \beta^{\prime}}-g^{\alpha \beta^{\prime}} g^{\beta \alpha^{\prime}}\right) \Pi\left(q^{2}\right),
\end{aligned}
$$

at both hadron and quark-gluon levels.

At the hadron level we use the dispersion relation to express Eq. (17) as

$$
\Pi\left(q^{2}\right)=\int_{0}^{\infty} \frac{\rho(s)}{s-q^{2}-i \varepsilon} d s,
$$


where $\rho(s) \equiv \operatorname{Im} \Pi(s) / \pi$ is the spectral density. We parametrize it using one pole dominance for the ground state $X$ together with the continuum contribution

$$
\begin{aligned}
\rho(s) & \equiv \sum_{n} \delta\left(s-M_{n}^{2}\right)\langle 0|J| n\rangle\left\langle n\left|J^{\dagger}\right| 0\right\rangle \\
& =f_{X}^{2} \delta\left(s-M_{X}^{2}\right)+\text { continuum. }
\end{aligned}
$$

At the quark-gluon level we insert $J_{1}^{\alpha \beta}$ into Eq. (17) and calculate it using the method of operator product expansion (OPE). Then we perform the Borel transformation to Eq. (18) at both hadron and quark-gluon levels. After approximating the continuum using the spectral density above a threshold value $s_{0}$, we obtain

$$
\Pi\left(s_{0}, M_{B}^{2}\right) \equiv f_{X}^{2} e^{-M_{X}^{2} / M_{B}^{2}}=\int_{0}^{s_{0}} e^{-s / M_{B}^{2}} \rho(s) d s,
$$

which can be used to calculate the mass of $X$ using

$$
M_{X}^{2}\left(s_{0}, M_{B}\right)=\frac{\int_{0}^{s_{0}} e^{-s / M_{B}^{2}} S \rho(s) d s}{\int_{0}^{s_{0}} e^{-s / M_{B}^{2}} \rho(s) d s} .
$$

In the present study we calculate OPEs up to the dimension eight $(D=8)$ condensates, including the perturbative term, the two-gluon condensate $\left\langle g_{s}^{2} G G\right\rangle \equiv\left\langle g_{s}^{2} G_{\mu \nu}^{a} G_{a}^{\mu \nu}\right\rangle$, the threegluon condensate $\left\langle g_{s}^{3} G^{3}\right\rangle \equiv\left\langle g_{s}^{3} f^{a b c} G_{a}^{\mu \nu} G_{b, \nu \rho} G_{c, \mu}^{\rho}\right\rangle$, and their combination $\left\langle g_{s}^{2} G G\right\rangle^{2}$ :

$$
\begin{aligned}
\rho_{1^{+-}}(s)= & \frac{4 \alpha_{s}^{3}}{81 \pi} s^{4}+\frac{10 \alpha_{s}^{2}\left\langle g_{s}^{2} G G\right\rangle}{9} s^{2}+\frac{35 \alpha_{s}^{3}\left\langle g_{s}^{2} G G\right\rangle}{36 \pi} s^{2} \\
& -\frac{205 \alpha_{s}^{2}\left\langle g_{s}^{3} G^{3}\right\rangle}{54} s, \\
\rho_{1^{--}}(s)= & \frac{4 \alpha_{s}^{3}}{81 \pi} s^{4}-\frac{10 \alpha_{s}^{2}\left\langle g_{s}^{2} G G\right\rangle}{9} s^{2}+\frac{25 \alpha_{s}^{3}\left\langle g_{s}^{2} G G\right\rangle}{36 \pi} s^{2} \\
& +\frac{5 \alpha_{s}^{2}\left\langle g_{s}^{3} G^{3}\right\rangle}{54} s, \\
\rho_{2^{+-}}(s)= & \frac{\alpha_{s}^{3}}{81 \pi} s^{4}+\frac{5 \alpha_{s}^{2}\left\langle g_{s}^{2} G G\right\rangle}{27} s^{2}+\frac{15 \alpha_{s}^{3}\left\langle g_{s}^{2} G G\right\rangle}{32 \pi} s^{2} \\
& -\frac{20 \alpha_{s}^{2}\left\langle g_{s}^{3} G^{3}\right\rangle}{27} s, \\
\rho_{2^{--}}(s)= & \frac{\alpha_{s}^{3}}{81 \pi} s^{4}-\frac{5 \alpha_{s}^{2}\left\langle g_{s}^{2} G G\right\rangle}{27} s^{2}+\frac{15 \alpha_{s}^{3}\left\langle g_{s}^{2} G G\right\rangle}{32 \pi} s^{2} \\
& -\frac{10 \alpha_{s}^{2}\left\langle g_{s}^{3} G^{3}\right\rangle}{27} s, \\
& +\frac{5 \alpha_{s}^{3}}{2016 \pi} s^{4}+\frac{\alpha_{s}^{2}\left\langle g_{s}^{2} G G\right\rangle}{16} s^{2}-\frac{59 \alpha_{s}^{3}\left\langle g_{s}^{2} G G\right\rangle}{512 \pi} s^{2} \\
& +\frac{13 \alpha_{s}^{2}\left\langle g_{s}^{3} G^{3}\right\rangle}{384} s, \\
\rho_{3^{+-}}(s) &
\end{aligned}
$$

$$
\begin{aligned}
\rho_{3^{--}}(s)= & \frac{5 \alpha_{s}^{3}}{2016 \pi} s^{4}-\frac{\alpha_{s}^{2}\left\langle g_{s}^{2} G G\right\rangle}{16} s^{2}-\frac{49 \alpha_{s}^{3}\left\langle g_{s}^{2} G G\right\rangle}{1536 \pi} s^{2} \\
& +\frac{157 \alpha_{s}^{2}\left\langle g_{s}^{3} G^{3}\right\rangle}{3456} s .
\end{aligned}
$$

Specifically, we find that all the $D=8$ terms proportional to $\left\langle g_{s}^{2} G G\right\rangle^{2}$ vanish, so the convergence of the above OPE series is quite good.

We need the strong coupling constant to perform numerical analyses [38]:

$$
\alpha_{s}\left(Q^{2}\right)=\frac{4 \pi}{11 \ln \left(Q^{2} / \Lambda_{\mathrm{QCD}}^{2}\right)},
$$

with the QCD scale at $\Lambda_{\mathrm{QCD}}=300 \mathrm{MeV}$.

We observed that the odderon mass $M_{X}$ depends significantly on the gluon condensate $\left\langle g_{s}^{2} G G\right\rangle$. However, this parameter is still not well known, so we use two sets of parameters:

(i) Parameter set-I [39]:

$$
\begin{aligned}
\left\langle\alpha_{s} G G\right\rangle & =(0.005 \pm 0.004) \times \pi \mathrm{GeV}^{4}, \\
\left\langle g_{s}^{3} G^{3}\right\rangle & =\left\langle\alpha_{s} G G\right\rangle \times(8.2 \pm 1.0) \mathrm{GeV}^{2} .
\end{aligned}
$$

(ii) Parameter set-II $[40,41]$ :

$$
\begin{aligned}
\left\langle\alpha_{s} G G\right\rangle & =(6.35 \pm 0.35) \times 10^{-2} \mathrm{GeV}^{4}, \\
\left\langle g_{s}^{3} G^{3}\right\rangle & =\left\langle\alpha_{s} G G\right\rangle \times(8.2 \pm 1.0) \mathrm{GeV}^{2} .
\end{aligned}
$$

As shown in Eq. (21), the mass $M_{X}$ depends on two free parameters, the threshold value $s_{0}$ and the Borel mass $M_{B}$. We use two criteria to determine the Borel window. Firstly, we investigate the convergence of the OPE, which is the cornerstone of a reliable sum rule analysis. Because the $D=8$ term proportional to $\left\langle g_{s}^{2} G G\right\rangle^{2}$ vanishes, this convergence is already quite good, but we further require the $\alpha_{s}^{n>3}$ terms $\alpha_{s}^{3}\left\langle g_{s}^{2} G G\right\rangle$ and $\alpha_{s}^{2}\left\langle g_{s}^{3} G^{3}\right\rangle$ to be less than or equal to $5 \%$ :

$$
\mathrm{CVG} \equiv\left|\frac{\Pi^{\alpha_{s}^{n>3}}\left(s_{0}, M_{B}^{2}\right)}{\Pi\left(s_{0}, M_{B}^{2}\right)}\right| \leq 5 \%
$$

Secondly, we investigate the one-pole-dominance assumption, and require the pole contribution (PC) to be greater than or equal to $40 \%$ :

$$
\mathrm{PC} \equiv\left|\frac{\Pi\left(s_{0}, M_{B}^{2}\right)}{\Pi\left(\infty, M_{B}^{2}\right)}\right| \geq 40 \% .
$$

Altogether we can determine the Borel window for a fixed $s_{0}$. Then we change $s_{0}$ and use the same procedures, so that we can find the lower bound of $s_{0}$. 
TABLE I. Masses of the $J^{P C}=1 / 2 / 3^{ \pm-}$odderons, extracted from the currents $J_{1 / 2 / 3}^{\alpha_{1} \cdots \alpha_{J}, \beta_{1} \cdots \beta_{J}}$ and $\tilde{J}_{1 / 2 / 3}^{\alpha_{1} \cdots \alpha_{J}, \beta_{1} \cdots \beta_{J}}$. In parameter set-I we choose the gluon condensate to be $\left\langle\alpha_{s} G G\right\rangle=(0.005 \pm 0.004) \times \pi \mathrm{GeV}^{4}$ [39], and in parameter set-II we choose it to be $\left\langle\alpha_{s} G G\right\rangle=$ $(6.35 \pm 0.35) \times 10^{-2} \mathrm{GeV}^{4}[40]$.

\begin{tabular}{|c|c|c|c|c|c|c|c|}
\hline & \multirow[b]{2}{*}{ Odderon } & \multirow[b]{2}{*}{ Current } & \multirow[b]{2}{*}{$s_{0}^{\min }\left[\mathrm{GeV}^{2}\right]$} & \multicolumn{2}{|c|}{ Working regions } & \multirow[b]{2}{*}{ Pole [\%] } & \multirow[b]{2}{*}{ Mass $[\mathrm{GeV}]$} \\
\hline & & & & $s_{0}\left[\mathrm{GeV}^{2}\right]$ & $M_{B}^{2}\left[\mathrm{GeV}^{2}\right]$ & & \\
\hline \multirow[t]{6}{*}{ Parameter set-I } & $X_{1^{+-}}$ & $J_{1}^{\alpha \beta}$ & 14.1 & $16.0 \pm 2.0$ & $3.67-4.13$ & $40-50$ & $2.87_{-0.20}^{+0.17}$ \\
\hline & $X_{2^{+-}}$ & $J_{2}^{\alpha_{1} \alpha_{2}, \beta_{1} \beta_{2}}$ & 10.6 & $16.0 \pm 2.0$ & $2.76-4.07$ & $40-73$ & $2.85_{-0.20}^{+0.16}$ \\
\hline & $X_{3^{+-}}$ & $J_{3}^{\alpha_{1} \alpha_{2} \alpha_{3}, \beta_{1} \beta_{2} \beta_{3}}$ & 8.9 & $16.0 \pm 2.0$ & $2.60-4.23$ & $40-81$ & $2.78_{-0.23}^{+0.18}$ \\
\hline & $X_{1^{--}}$ & $\tilde{J}_{1}^{\alpha \beta}$ & 15.1 & $17.0 \pm 2.0$ & $2.93-3.52$ & $40-54$ & $3.29_{-0.32}^{+1.49}$ \\
\hline & $X_{2^{--}}$ & $\tilde{J}_{2}^{\alpha_{1} \alpha_{2}, \beta_{1} \beta_{2}}$ & 15.3 & $17.0 \pm 2.0$ & $3.29-3.74$ & $40-50$ & $3.16_{-0.23}^{+0.33}$ \\
\hline & $X_{3^{--}}$ & $\tilde{J}_{3}^{\alpha_{1} \alpha_{2} \alpha_{3}, \beta_{1} \beta_{2} \beta_{3}}$ & 15.0 & $17.0 \pm 2.0$ & $2.55-3.36$ & $40-58$ & $3.47_{-0.50}^{+?}$ \\
\hline \multirow[t]{6}{*}{ Parameter set-II } & $X_{1^{+-}}$ & $J_{1}^{\alpha \beta}$ & 19.0 & $21.0 \pm 2.0$ & $5.38-5.88$ & $40-47$ & $3.27_{-0.17}^{+0.15}$ \\
\hline & $X_{2^{+-}}$ & $J_{2}^{\alpha_{1} \alpha_{2}, \beta_{1} \beta_{2}}$ & 12.6 & $21.5 \pm 2.0$ & $3.64-5.84$ & $40-79$ & $3.28_{-0.19}^{+0.14}$ \\
\hline & $X_{3^{+-}}$ & $J_{3}^{\alpha_{1} \alpha_{2} \alpha_{3}, \beta_{1} \beta_{2} \beta_{3}}$ & 19.8 & $22.0 \pm 2.0$ & $5.77-6.27$ & $40-47$ & $3.30_{-0.17}^{+0.15}$ \\
\hline & $X_{1^{--}}$ & $\tilde{J}_{1}^{\alpha \beta}$ & 32.6 & $35.0 \pm 3.0$ & $6.05-6.93$ & $40-49$ & $5.05_{-0.14}^{+0.17}$ \\
\hline & $X_{2^{--}}$ & $\tilde{J}_{2}^{\alpha_{1} \alpha_{2}, \beta_{1} \beta_{2}}$ & 29.1 & $36.0 \pm 3.0$ & $5.96-7.93$ & $40-63$ & $4.72_{-0.17}^{+0.15}$ \\
\hline & $X_{3^{--}}$ & $\tilde{J}_{3}^{\alpha_{1} \alpha_{2} \alpha_{3}, \beta_{1} \beta_{2} \beta_{3}}$ & 34.5 & $37.0 \pm 3.0$ & $5.90-7.07$ & $40-50$ & $5.45_{-0.21}^{+0.32}$ \\
\hline
\end{tabular}

Take the current $J_{1}^{\alpha \beta}$ as an example. When using the parameter set-I, nonvanishing Borel windows exist as long as $s_{0}>14.1 \mathrm{GeV}^{2}$, and the Borel window is determined to be $3.67 \mathrm{GeV}^{2}<M_{B}^{2}<4.13 \mathrm{GeV}^{2}$ for $s_{0}=16 \mathrm{GeV}^{2}$. Accordingly, we choose the working regions to be $14.0 \mathrm{GeV}^{2}<s_{0}<18.0 \mathrm{GeV}^{2}$ and $3.67 \mathrm{GeV}^{2}<M_{B}^{2}<$ $4.13 \mathrm{GeV}^{2}$, and calculate the mass of the $1^{+-}$odderon to be

$$
M_{X_{1^{+-}}}=2.87_{-0.20}^{+0.17} \mathrm{GeV}
$$

Its uncertainty is quite large, mainly stemming from the uncertainty of the gluon condensate $\left\langle g_{s}^{2} G G\right\rangle$ given in Eqs. (29).

Similarly, we use the currents $J_{1 / 2 / 3}^{\alpha_{1} \cdots \alpha_{J}, \beta_{1} \cdots \beta_{J}}$ and $\tilde{J}_{1 / 2 / 3}^{\alpha_{1} \cdots \alpha_{J}, \beta_{1} \cdots \beta_{J}}$ to perform numerical analyses, and calculate masses of the $J^{P C}=1 / 2 / 3^{ \pm-}$odderons. The obtained results are summarized in Table I. Note that the OPE convergence is sometimes so good that the lower bound of $M_{B}$ cannot be well determined, and in such cases we need to properly choose $s_{0}$ according to their partner states, e.g., see the result of $X_{2^{+-}}$using the parameter set-II. We clearly see from Table I that our QCD sum rule results depend significantly on the gluon condensate $\left\langle g_{s}^{2} G G\right\rangle$, which is currently not well known and understood and is still waiting to be clarified.

\section{SUMMARY AND DISCUSSIONS}

In this article we apply the method of QCD sum rules to study the odderon as a three-gluon bound state. There may exist six lowest-lying odderons with the quantum numbers
$J^{P C}=1 / 2 / 3^{ \pm-}$. We systematically construct their interpolating currents using the gluon field strength tensors $G_{\mu \nu}^{a}$ and $\tilde{G}_{\mu \nu}^{a}$. All these currents have $N=2 J$ Lorentz indices with certain symmetries, so that they couple to both the positive- and negative-parity odderons, which need to be further separated at the hadron level. The construction of such currents is quite general and may be applied in fields other than hadron physics.

We constructed six relativistic low-dimension odderon currents with the quantum numbers $J^{P C}=1 / 2 / 3^{ \pm-}$. We used them to perform QCD sum rule analyses, and calculated the masses of the $J^{P C}=1 / 2 / 3^{ \pm-}$odderons. The results are summarized in Table I, sometimes with quite large uncertainties coming from the gluon condensates $\left\langle g_{s}^{2} G G\right\rangle$ and $\left\langle g_{s}^{3} G^{3}\right\rangle$. It is interesting to compare our results with the lattice QCD results [21-24] obtained using nonrelativistic odderon operators, as given in Table II.

From the above comparison, we can see how poorly we understand the odderon. Recall that there is currently no definite experimental evidence for the existence of any glueball, and we realize the importance of the evidence of the odderon exchange recently observed by D0 and TOTEM [2]. Since this is still an indirect evidence, we propose a direct search for the odderon using the LHC.

Using the quark model, the odderon can decay after exciting three quark-antiquark pairs, and recombine into three mesons. Generally speaking, its width can be quite large, preventing it from being easily observed. We use $P$ and $V$ to denote the light vector and pseudoscalar mesons respectively, and its possible decay patterns are 
TABLE II. Masses of the $J^{P C}=1 / 2 / 3^{ \pm-}$odderons, in units of GeV. Our QCD sum rule results are obtained using parameter set-I and parameter set-II, and the lattice QCD results are taken from Refs. [21-23] (quenched) and Ref. [24] (unquenched).

\begin{tabular}{|c|c|c|c|c|c|c|}
\hline Odderon & Set-I & Set-II & Ref. [21] & Ref. [22] & Ref. [23] & Ref. [24] \\
\hline$X_{1^{+-}}$ & $2.87_{-0.20}^{+0.17}$ & $3.27_{-0.17}^{+0.15}$ & $2.98 \pm 0.03 \pm 0.14$ & $2.94 \pm 0.03 \pm 0.14$ & $2.67 \pm 0.07 \pm 0.12$ & $3.27 \pm 0.34$ \\
\hline$X_{2^{+-}}$ & $2.85_{-0.20}^{+0.16}$ & $3.28_{-0.19}^{+0.14}$ & $4.23 \pm 0.05 \pm 0.20$ & $4.14 \pm 0.05 \pm 0.20$ & $\cdots$ & $\cdots$ \\
\hline$X_{3^{+-}}$ & $2.78_{-0.23}^{+0.18}$ & $3.30_{-0.17}^{+0.15}$ & $3.60 \pm 0.04 \pm 0.17$ & $3.55 \pm 0.04 \pm 0.17$ & $3.27 \pm 0.09 \pm 0.15$ & $3.85 \pm 0.35$ \\
\hline$X_{1^{--}}$ & $3.29_{-0.32}^{+1.49}$ & $5.05_{-0.14}^{+0.17}$ & $3.83 \pm 0.04 \pm 0.19$ & $3.85 \pm 0.05 \pm 0.19$ & $3.24 \pm 0.33 \pm 0.15$ & $\cdots$ \\
\hline$X_{2^{--}}$ & $3.16_{-0.23}^{+0.33}$ & $4.72_{-0.17}^{+0.15}$ & $4.01 \pm 0.05 \pm 0.20$ & $3.93 \pm 0.04 \pm 0.19$ & $3.66 \pm 0.13 \pm 0.17$ & $4.59 \pm 0.74$ \\
\hline$X_{3^{--}}$ & $3.47_{-0.50}^{+? .25}$ & $5.45_{-0.21}^{+0.32}$ & $4.20 \pm 0.05 \pm 0.20$ & $4.13 \pm 0.09 \pm 0.20$ & $4.33 \pm 0.26 \pm 0.20$ & \\
\hline
\end{tabular}

$$
\begin{aligned}
1^{--} & \rightarrow V P P, V V P, V V V(S \text {-wave }) \\
1^{+-} & \rightarrow P P P, V P P, V V P, V V V(P \text {-wave }), \\
2^{--} & \rightarrow V V P, V V V(S \text {-wave }) \\
2^{+-} & \rightarrow V P P, V V P, V V V(P \text {-wave }), \\
3^{--} & \rightarrow V V V(S \text {-wave }) \\
3^{+-} & \rightarrow V V P, V V V(P \text {-wave }) .
\end{aligned}
$$

Due to their limited decay patterns, the spin-3 odderons probably have relatively small widths, and so we propose to search for them in their $V V V$ and $V V P$ decay channels directly using the LHC.

\section{ACKNOWLEDGMENTS}

S. L. Z is grateful to Shou-Hua Zhu for helpful discussions. This project is supported by the National Natural Science Foundation of China under Grants No. 11722540, No. 11975033, No. 12075019, and No. 12070131001, the National Key Research and Development Program of China (2020YFA0406400), and the Fundamental Research Funds for the Central Universities.
[1] V. M. Abazov et al. (D0 Collaboration), Measurement of the differential cross section $d \sigma / d t$ in elastic $p \bar{p}$ scattering at $\sqrt{s}=1.96 \mathrm{TeV}$, Phys. Rev. D 86, 012009 (2012).

[2] V. M. Abazov et al. (D0 and TOTEM Collaborations), Comparison of $p p$ and $p \bar{p}$ differential elastic cross sections and observation of the exchange of a colorless $C$-odd gluonic compound, arXiv:2012.03981.

[3] J. R. Cudell, V. V. Ezhela, P. Gauron, K. Kang, Yu. V. Kuyanov, S. B. Lugovsky, E. Martynov, B. Nicolescu, E. A. Razuvaev, and N. P. Tkachenko (COMPETE Collaboration), Benchmarks for the Forward Observables at RHIC, the Tevatron-Run II and the LHC, Phys. Rev. Lett. 89, 201801 (2002).

[4] E. Martynov and B. Nicolescu, Odderon effects in the differential cross-sections at Tevatron and LHC energies, Eur. Phys. J. C 79, 461 (2019).

[5] G. Antchev et al. (TOTEM Collaboration), First determination of the $\rho$ parameter at $\sqrt{s}=13 \mathrm{TeV}$ : Probing the existence of a colorless $C$-odd three-gluon compound state, Eur. Phys. J. C 79, 785 (2019).

[6] V. A. Khoze, A. D. Martin, and M. G. Ryskin, Black disk, maximal Odderon and unitarity, Phys. Lett. B 780, 352 (2018).

[7] T. Csörgő, R. Pasechnik, and A. Ster, Odderon and proton substructure from a model-independent Lévy imaging of elastic $p p$ and $p \bar{p}$ collisions, Eur. Phys. J. C 79, 62 (2019).
[8] V. P. Gonçalves and P. V. R. G. Silva, The Phillips-Barger model for the elastic cross section and the odderon, Eur. Phys. J. C 79, 237 (2019).

[9] W. Xie, A. Watanabe, and M. Huang, Elastic proton-proton scattering at LHC energies in holographic QCD, J. High Energy Phys. 10 (2019) 053.

[10] L. Lukaszuk and B. Nicolescu, A possible interpretation of $p p$ rising total cross-sections, Lett. Nuovo Cimento 8, 405 (1973).

[11] J. Bartels, High-energy behavior in a non-Abelian gauge theory (II). First corrections to $T_{n \rightarrow m}$ beyond the leading $\ln s$ approximation, Nucl. Phys. B175, 365 (1980).

[12] J. Kwiecinski and M. Praszalowicz, Three gluon integral equation and odd $\mathrm{C}$ singlet Regge singularities in QCD, Phys. Lett. 94B, 413 (1980).

[13] A. Donnachie and P. V. Landshoff, Multi-gluon exchange in pp elastic scattering, Phys. Lett. 123B, 345 (1983).

[14] M. M. Block, Hadronic forward scattering: Predictions for the Large Hadron Collider and cosmic rays, Phys. Rep. 436, 71 (2006).

[15] A. Chodos, R. L. Jaffe, K. Johnson, C. B. Thorn, and V. F. Weisskopf, New extended model of hadrons, Phys. Rev. D 9, 3471 (1974).

[16] R. L. Jaffe and K. Johnson, Unconventional states of confined quarks and gluons, Phys. Lett. 60B, 201 (1976).

[17] N. Isgur and J. E. Paton, Flux-tube model for hadrons in QCD, Phys. Rev. D 31, 2910 (1985). 
[18] A. Szczepaniak, E. S. Swanson, C. R. Ji, and S. R. Cotanch, Glueball Spectroscopy in a Relativistic Many-Body Approach to Hadron Structure, Phys. Rev. Lett. 76, 2011 (1996).

[19] F. J. Llanes-Estrada, P. Bicudo, and S. R. Cotanch, $J^{--}$ Glueballs and a Low Odderon Intercept, Phys. Rev. Lett. 96, 081601 (2006).

[20] K. G. Wilson, Confinement of quarks, Phys. Rev. D 10, 2445 (1974).

[21] Y. Chen et al., Glueball spectrum and matrix elements on anisotropic lattices, Phys. Rev. D 73, 014516 (2006).

[22] V. Mathieu, N. Kochelev, and V. Vento, The physics of glueballs, Int. J. Mod. Phys. E 18, 1 (2009).

[23] H. B. Meyer, Glueball regge trajectories, hep-lat/0508002.

[24] E. Gregory, A. Irving, B. Lucini, C. McNeile, A. Rago, C. Richards, and E. Rinaldi, Towards the glueball spectrum from unquenched lattice QCD, J. High Energy Phys. 10 (2012) 170.

[25] V. A. Novikov, M. A. Shifman, A. I. Vainshtein, and V. I. Zakharov, In search of scalar gluonium, Nucl. Phys. B165, 67 (1980).

[26] S. Narison, Spectral function sum rules for gluonic currents, Z. Phys. C 26, 209 (1984).

[27] S. Narison, Masses, decays and mixings of gluonia in QCD, Nucl. Phys. B509, 312 (1998).

[28] E. Bagan and T. G. Steele, Mass of the scalar glueball. Higher-loop effects in the QCD sum rules, Phys. Lett. B 243, 413 (1990).

[29] J. I. Latorre, S. Narison, and S. Paban, $0^{++}$trigluonium sum rules, Phys. Lett. B 191, 437 (1987).
[30] G. Hao, C. F. Qiao, and A. L. Zhang, $0^{-+}$trigluon glueball and its implication for a recent BES observation, Phys. Lett. B 642, 53 (2006).

[31] C. F. Qiao and L. Tang, Finding the $0^{--}$Glueball, Phys. Rev. Lett. 113, 221601 (2014).

[32] L. Tang and C. F. Qiao, Mass spectra of $0^{+-}, 1^{-+}$, and $2^{+-}$ exotic glueballs, Nucl. Phys. B904, 282 (2016).

[33] A. Pimikov, H. J. Lee, N. Kochelev, and P. Zhang, Is the exotic $0^{--}$glueball a pure gluon state? Phys. Rev. D 95, 071501(R) (2017).

[34] I. Szanyi, L. Jenkovszky, R. Schicker, and V. Svintozelskyi, Pomeron/glueball and odderon/oddball trajectories, Nucl. Phys. A998, 121728 (2020).

[35] R. L. Jaffe, K. Johnson, and Z. Ryzak, Qualitative features of the glueball spectrum, Ann. Phys. (N.Y.) 168, 344 (1986).

[36] M. A. Shifman, A. I. Vainshtein, and V. I. Zakharov, QCD and resonance physics. theoretical foundations, Nucl. Phys. B147, 385 (1979).

[37] L. J. Reinders, H. Rubinstein, and S. Yazaki, Hadron properties from QCD sum rules, Phys. Rep. 127, 1 (1985).

[38] P. A. Zyla et al. (Particle Data Group), Review of particle physics, Prog. Theor. Exp. Phys. 2020, 083 C01 (2020).

[39] B. L. Ioffe, QCD (Quantum chromodynamics) at low energies, Prog. Part. Nucl. Phys. 56, 232 (2006).

[40] S. Narison, QCD parameter correlations from heavy quarkonia, Int. J. Mod. Phys. A 33, 1850045 (2018); Addendum, Int. J. Mod. Phys. A 33, 1892004 (2018).

[41] S. Narison, Gluon condensates and precise $\bar{m}_{c, b}$ from QCDmoments and their ratios to order $\alpha_{s}^{3}$ and $\left\langle G^{4}\right\rangle$, Phys. Lett. B 706, 412 (2012). 